\title{
SEIZURE SOURCE SELECTION VIA A HYBRID SECOND ORDER BLIND IDENTIFICATION AND GAP STATISTIC ALGORITHM
}

\author{
${ }^{1}$ Delaram Jarchi, ${ }^{2}$ Reza Boostani, and ${ }^{3}$ Saeid Sanei \\ ${ }^{1.2}$ Department of Computer Science and Engineering, Shiraz University, Shiraz, Iran \\ ${ }^{3}$ Centre of DSP, School of Engineering, Cardiff University, Cardiff, U.K.
}

\begin{abstract}
In this paper a new method for isolating main seizure source from multichannel scalp EEG is proposed. First, the number of active brain sources in each time frame is determined. Then, Second Order Blind Identification (SOBI) is applied to isolate brain sources in a number of signal segments. After applying SOBI to the signals during seizure, rows of resulted unmixing matrix are clustered by k-means clustering method. The value of $\mathrm{k}$ is estimated via gap statistic method. By multiplying each cluster center to the electrode signals, the brain sources are extracted. The results showed that seizure source could be effectively separated from other brain sources.
\end{abstract}

Index Terms - Seizure, SOBI, BSS, k-means, Gap statistic.

\section{INTRODUCTION}

Epilepsy is the most common neurological disorder, second only to stroke. Nearly 60 million people worldwide are diagnosed with epilepsy whose hallmark is recurrent seizures [8].[9]. EEG is the most commonly used data for the evaluation of brain activity and neurological abnormalities [1]. Most analysis of epilepsy is based on the EEG. Recorded raw EEG signals are mixture of underlying sources within brain. An effective way to obtain brain source signals is necessary in order to analyze EEG signals. Several Blind Source Separation (BSS) algorithms are applied to the raw EEG signals to extract main brain sources such as Infomax algorithm [5] and JADE algorithm [6]. Most BSS algorithms assume that the EEG sources have non-Gaussian distribution, However, the actual statistics of these sources within the brain are yet unknown [1]. In this paper, the well known SOBI algorithm is used to extract the source signals, mainly because the SOBI makes fewer assumptions about the statistical properties of the data than other BSS algorithms [1].

In places where the number of sources is unknown a common practice in BSS is to consider similar number of sources and mixtures. However, when the number of EEG recording electrodes is reasonably high it is expected to have a fewer number of sources than sensors, where some of the estimated sources are likely to be noise and artifacts. Here, we estimate the number of active brain signal sources by the algorithm proposed by Xiaoxiao Bai et al. [2]. Hence, the number of sources in the proposed BSS is considered known. Since it is needed to apply SOBI to a number of time frames with slight overlaps, a relation must be established between the estimated sources in consecutive time frames. A clustering method on rows of unmixing matrix is followed to cluster each row. It is clear that in focal seizures of the same patient the seizure foci remains fixed. Hence by clustering the column vectors we can point to the center of that cluster which relates to a seizure source, as a way for localization of seizure source. Other brain sources can be obtained by simply considering the estimated cluster centers. Optimal number of clusters is estimated via gap statistic method. Extracting the seizure source is necessary for further analysis such as localization of seizure. Our methodology is performed in two stages; first applying BSS method (here, SOBI), then clustering the common spatial patterns. The remainder of this paper is structured as follows:

In section 2, estimation of the number of independent sources within the brain is described. In section 3 , the SOBI algorithm is briefly described and in section 4, gap statistic method is described, then in section 5 , our proposed method followed by some experimental results is explained.

\section{ESTIMATION OF NUMBER OF INDEPENDENT SOURCES}

The brain sources are mixed and recorded as the scalp EEG. $\mathbf{x}(t)=\left[x_{1}(t), \cdots, x_{m}(t)\right]^{T}[2]$, where $m$ denotes the number of electrodes and $(.)^{T}$ represents transpose operation. Assume that we have $m$ electrodes, thus each $\mathbf{x}(t)$ is a vector of size $\boldsymbol{m} \times 1$. The data matrix $\mathbf{X}$ is a $\boldsymbol{m} \times \boldsymbol{T}$ matrix (where $\boldsymbol{T}$ is the number of time points).

The procedure to detect the number of independent sources of EEG signals is briefly described in the following steps:

Step1. Calculating the covariance matrix $\mathrm{C}$ from the measured data matrix $\mathbf{X}$. 
Step2. Using Singular value decomposition (SVD) to decompose the covariance matrix $\mathrm{C}$, and calculate all the eigenvalues considering that $\lambda_{1}>\ldots>\lambda_{m}$.

Step3. Calcaulating Information Criterion (IC) value with cigenvalues of the covariance matrix $\mathrm{C}$ base on formula given below:

$$
\begin{aligned}
& I C=-\left(T-1-k-\frac{2(m-k)^{2}+m-k+2}{6(m-k)}\right. \\
& \left.+\sum_{i=1}^{k} \frac{\bar{\lambda}_{m-k}^{2}}{\left(\lambda_{i}-\bar{\lambda}_{m-k}\right)^{2}}\right) \cdot \log \left(\bar{\lambda}_{m-k}^{-(m-k)} \prod_{i=k+1}^{m} \lambda_{i}\right) \\
& -2 d(k, m) C_{f}(T-1)
\end{aligned}
$$

Where $d(k, m)=k(m-k+2)(m-k-1) / 2, k$ is number of assumed dipole sources and $\bar{\lambda}_{m-k}$ is the average of $m-k$ smallest eigenvalues. $C_{f}(h)(h=T$ or $h=T-1)$ is the penalty function that can be one of the followings:
1) $C_{1}=2$
2) $C_{2}=2 \log (\log (h))$
3) $C_{3}=\log (h)$
4) $C_{4}=2 \log (h)$
5) $C_{5}=3 \log (h)$

For each number from 1 to $k$ ( $k$ is number of assumed sources), related Information Criterion must be calculated $\left(I C_{k}\right)$.

Step 4. The number of sources that have minimum IC will be selected as the estimated number of sources.

\section{SECOND ORDER BLIND IDENTIFICATION}

Second Order Blind Identification (SOBI) is able to estimate mixing matrix as long as the unknown source signals are temporally uncorrelated to each other but they have nonzero time-delayed autocorrelations. This is a plausible assumption for the case of EEG and EOG sources.

SOBI computes the mixing matrix as the matrix that jointly diagonalizes [7] a set of $p$ cross correlation matrices $R\left(\tau_{i}\right)=E\left[x(t) x\left(t-\tau_{i}\right)^{T}\right]$, where $i=1, \ldots, p$, and $E[$. is the expectation operator. In this paper we use $p$ - $\left\lfloor L_{a} / 3\right\rfloor$ where $L_{a}$ is the number of EEG data samples in the analyzed data frame and $L$. $\rfloor$ denotes the floor operator.

\section{GAP STATISTIC}

Gap statistic is a method for estimating the number of clusters in a set of data. This technique uses the output of any clustering algorithm such as k-means and compares the change in within cluster dispersion to that expected under a uniform null distribution [3].

In this section, computation of the Gap statistic is briefly described as follows:

Step 1. Cluster the observed data, varying the total number of clusters from $k=1,2, \ldots, K$, So we have $K$ clusters $C_{1}, C_{2}, \ldots, C_{K}$. The pairwise distance within the cluster $C_{i}$ is given by :

$$
\sum_{j, j^{\prime} \in C_{i}}\left\|a_{j}-a_{j^{\prime}}\right\|_{2}^{2}
$$

where $a_{j}$ and $a_{j^{\prime}}$ are vectors in cluster $\mathrm{i}^{\text {th }}$. within dispersion measures $W_{k}, k=1,2, \ldots, K$ can be calculated via the following formula:

$$
W_{k}=\sum_{i=1}^{k} \frac{1}{2 n_{i}} \sum_{j, j^{\prime} \in C_{i}}\left\|a_{j}-a_{j^{\prime}}\right\|_{2}^{2}
$$

where, $n_{i}$ is the number of vectors within the $\mathrm{i}^{\text {th }}$ cluster.

Step 2. Generate B reference datasets, using the choice a or $b$ that is described below, and cluster each one giving dispersion measures $W_{k b}^{*}, b=1,2, \ldots, \mathrm{B}, k=1,2, \ldots, K$. Compute the estimated Gap statistic:

$$
\mathrm{Gap}(k)-(1 / \mathrm{B}) \sum_{b=1}^{B} \log \left(W_{k b}^{*}\right)-\log \left(W_{k}\right)
$$

There are different ways the reference data distribution can be selected:

(a) Generate each reference feature uniformly over the range of the observed values for that feature.

(b) Generate the reference features from a uniform distribution over a box aligned in the directions of the principle components of the data. In detail, if $\mathrm{X}$ is the $\boldsymbol{n} \times \boldsymbol{p}$ data matrix, assume the columns have zero means and compute the singular value decomposition $\mathrm{X}=\mathrm{UDV}^{\mathrm{T}}$. Transform the data via $\mathrm{X}^{\prime}=\mathrm{XV}$ and then draw uniform 
features $Z^{\prime}$ over the range of the columns of $X^{\prime}$, as in step (a) above.

(c) Finally back transform via $Z=Z^{\prime} V^{T}$ to obtain the reference data $Z$.

Step 3. Let $\bar{l}=(1 / B) \sum_{b=1}^{B} \log \left(W_{k b}^{*}\right)$, compute the standard deviation $s d_{k}=\left[(1 / B) \sum_{b=1}^{B}\left(\log \left(W_{k b}^{*}\right)-\bar{l}\right)^{2}\right]^{\frac{1}{2}}$, and define $S_{k}=s d_{k} \sqrt{1+1 / B}$. Finally choose the number of clusters via:

$\hat{k}=$ smallest $k$ such that

$$
G a p(k) \geq G a p(k+1)-s_{k+1}
$$

\section{EXPERIMENTAL RESULTS}

Here, we combined SOBI algorithm with Gap statistics approach to separate the EEG signals and detect the source of interest i.e. epileptic seizure source. Gap statistics in connection with k-means algorithm cluster the column vectors of the unmixing matrix driven by SOBI BSS algorithm. On the other hand the EEG source is identified in places where short-term largest Lyapunov exponent $\left(S T L_{\max }\right.$ ), the nonlinear dynamical measure that contains local information about dynamical mixing in the brain [4] , has its minimum value often after a down trend in the measurement of Lyaponuv exponents.

Moreover, the number of independent brain sources is estimated by the method described in section 2 before applying BSS. This makes the overall BSS system evendetermined

In this research scalp EEG signals of 4 patients with focal seizure are examined. Each EEG recordings takes 2 minutes long (sample frequency of $200 \mathrm{~Hz}$ ) from 16 channels. For each subject, $S T L_{\max }$ is used to estimate approximately the seizure onset. Thus, $S T L_{\max }$ measurement has been performed for the different channels of scalp signals. The parameters used here, is the same as the parameters for estimating $S T L_{\max }$ in [4].

As the signal length is limited, to have more $S T L_{\max }$ to better understand the complexity of the system, the segments of duration of 12 seconds with $80 \%$ overlap were used. The estimated $S T L_{\max }$ values of channel 1 is depicted in Figure 1.

The estimated $S T L_{\max }$ values of channel 2 is nearly the same as channel 1 except that the minimum value of
$S T L_{\max }$ is a little before $60^{\text {th }}$ second. Therefore, it is considered seizure onset about $60^{\text {th }}$ second. Scalp signals of all channels from $40^{\text {th }}$ second to $80^{\text {th }}$ second is divided into 8 segments with $10 \%$ overlap. Resulted number of sources by the method described in section 2 is as follows:

$$
8,8,8,10,10,12,13,13
$$

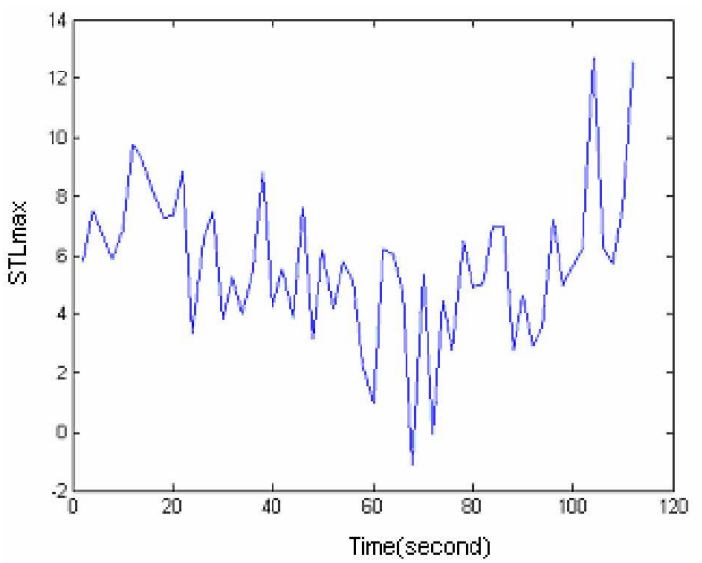

Figure 1. Estimated $S T L_{\max }$ values of channel 1 with $80 \%$ overlapped segments.

As it is expected, the number of sources from one frame to another varies. SOBI with the given number of sources in each time frame is applied to all the eight frames. Then Gap statistic method is applied to rows of resulted unmixing matrix of all frames. Number of data clusters is estimated 8 by gap statistic method. It is used k-means clustering with $\mathrm{k}=8$ to cluster rows of all 8 unmixing matrix. After clustering each cluster center is used to extract a source. Each cluster center is multiplied by scalp electrodes to get estimated sources. Our main interest is to extract seizure source, and as it is clear that for a focal seizure, seizure remains in the same place, cluster center of seizure source can give us the main seizure source. Resulted sources of the explained method is shown in Figure 2. Maximum short term Lyapunov exponents has been measured from the second signal of Figure 2 (from top). The size of data segment duration is 10 seconds with $80 \%$ overlap. These measures are depicted in Figure 3. From the figure It is clear that there is a drop in $\mathrm{STL}_{\max }$ values around 14 seconds. So we can consider this signal as the main seizure signal. As it is said that our segments starts from 40 seconds of the main electrode signals. We can simply add 40 to 14 to find approximate seizure onset. Hence, second 54 corresponds to seizure onset. This value seems reasonable as we expected seizure onset about to be at 60 seconds, because of the $\mathrm{STL}_{\text {max }}$ values that obtained from channel 1 and channel 2. 


\section{CONCLUSIONS}

In this paper a new two-stage method for extracting the seizure signal source from the scalp EEGs is proposed. Seizure source plays an important role in analyzing seizure activity and determining seizure onset. It also contributes to prediction of seizure and seizure localization in the case of focal seizures.

The procedure to find the number of brain sources in each time frame, used in this paper is very effective to find the relevant sources. In future researches, some improvements can be done on clustering method and adopt other ideas in the clustering field like rival penalized competitive learning RPCL) and also on implementing constrained BSS techniques to incorporate physiological information.
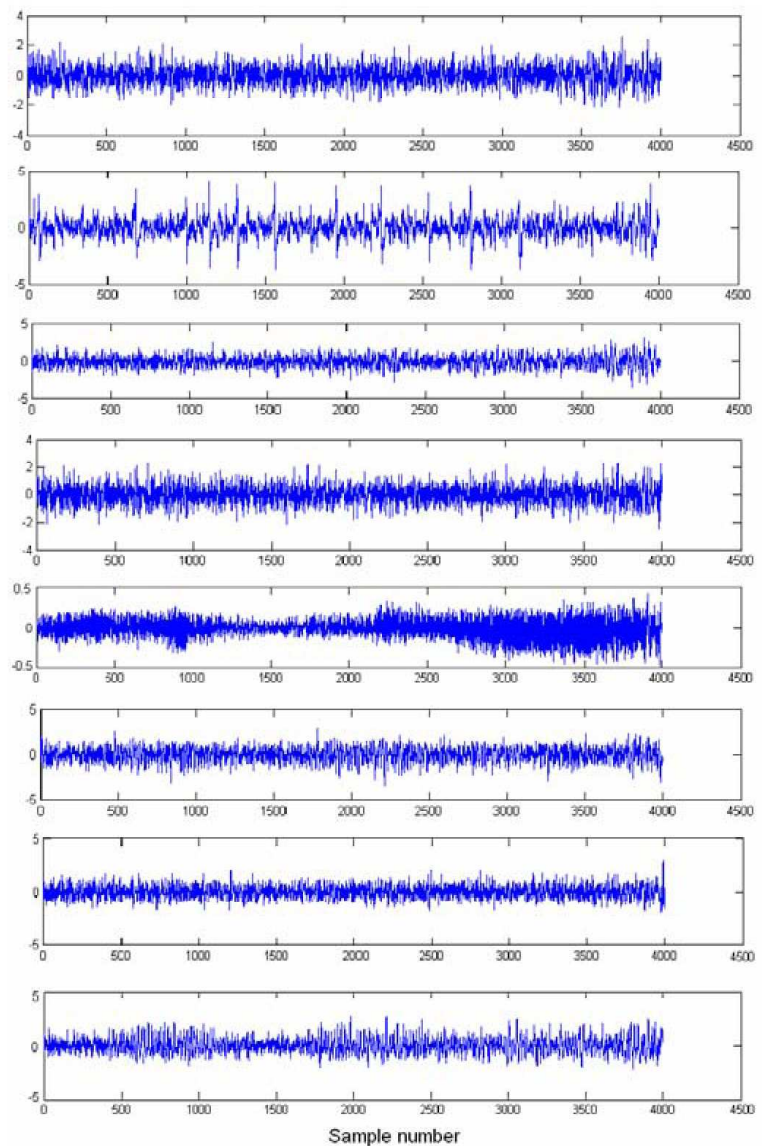

Figure 2. Estimated EEG sources of a patient with focal seizure.

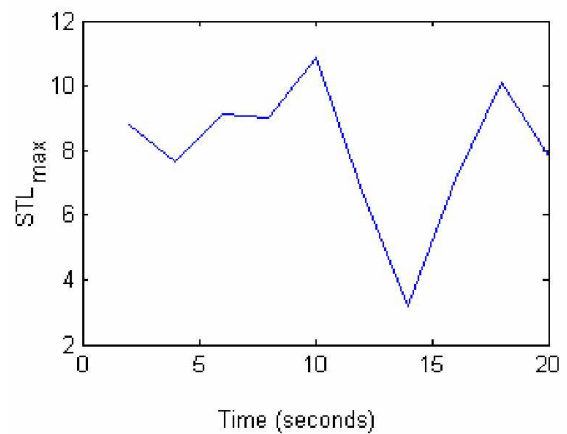

Figure 3. Estimated $\mathrm{STL}_{\max }$ values for the second signal in Figure 2.

\section{REFERENCES}

[1] J. Corsini, L. Shoker, S. Sanei, and G. Alarcón," Epileptic Seizure Predictability From Scalp EEG Incorporating Constrained Blind Source Separation," IEEE Transactions on biomedical Engineering, vol. 53, No. 5, May 2006

[2] X. Bai and B. He "Estimation of Number of Independent Brain ElectricSources From the Scalp EEGs," IEEE Trans. on biomedical Engineering, vol. 53, No. 10, October 2006.

[3] R Tibshuani, G. Walther, and T. Hastie. Estimating the number of clusters in a dataset via the gap statistic.Tech. report, Stanford UniversiB Published in JRSSB, 2000.

[4] L. D. Iasemidis, J. C. Principe, and J. C. Sackellares, "Measurement and quantification of spatio-temporal dynamics of human epileptic seizures," in Nonlinear Biomedical Signal Processing, M. Akay, Ed. Piscataway, NJ: IEEE Press, 2000.

[5] A. J. Bell and T. J. Sejnowski, "An information-maximization approach to blind separation and blind deconvolution," Neural Computation, vol. 7, pp. 1004-1034, 1995.

[6] J.-F. Cardoso and A. Souloumiac, "Blind beamforming for non Guassian signals," Inst. Elect. Eng. Proc. $F$, vol. 140, pp. 362-370, 1993.

[7] J.-F. Cardoso and A. Souloumiac, "Jacobi angles for simultaneous diagonalization," SIAM J. Matrix Anal. Appl., vol. 17, pp. 161-164, 1996.

[8] L. D. Iasemidis, "Epileptic seizure prediction and control," IEEE Trans. Biomed. Eny., vol. 50, no. 5, pp. 549-558, 2003.

[9] S. Sanei and J. Chambers, EEG Signal Processing, John Wiley, NY, 2007. 